\title{
Application of Symmetric Orthogonal Multiwavelets and Prefilter Technique For Image Compression
}

\author{
Jiazhong Chen, Jingli Zhou, Shengsheng Yu, Qian Xiao, and Jun Xu \\ Computer College, Huazhong University of Science \& Technology, Wuhan, Hubei 430074, P. R. China
}

\begin{abstract}
Multiwavelets are new addition to the body of wavelet theory. There are many types of symmetric multiwavelets such as Geronimo-Hardin-Massopust (GHM) and Chui-Lian (CL) multiwavelets. However, the matrix filter generating the GHM system multiwavelets does not satisfy the symmetric property. For this reason, this paper presents a new method to construct the symmetric orthogonal matrix filter, which leads to the symmetric orthogonal multiwavelets (SOM). Moreover, we analyze the prefilter technique, corresponding to the symmetric orthogonal matrix filter, to get a good combining frequency response. To prove the good property of SOM in image compression application, we compared the compression effect with other writers' work, which was in published literature.
\end{abstract}

Key words: Image compression, Multiwavelets, Prefilter technique.

\section{INTRODUCTION}

Multiwavelets have several advantages in comparison with scalar wavelets. The features such as compact support, orthogonality, symmetry, and high order vanish moments are known to be important in signal processing. A scalar wavelet can not possess all these properties at the same time but multiwavelets can.

The study of multiwavelets was initiated by Goodman, Lee and Tang in [1]. Then Goodman and Lee in [2] discovered the characterization of scaling functions wavelets. In [3], Jia constructed a class of continuous orthogonal double wavelets with symmetry, short support, and orthogonality. The special case of [3] with multiplicity 2 and support [0, 2], was studied by Chui and Lian [4]. In [5], Hong and $\mathrm{Wu}$ constructed a class of multiwavelets with multiplicity 4 and support $[0,2]$. Generally, after the presentation of prefilter technique, multiwavelets with multiplicity 2 can be applied in image compression application successfully [6][7][8].
The matrix filter generating the GHM, is not symmetric. So GHM can not solve the edge problem accurately in image coding unless the matrix filter used to do transform is symmetric. Though the matrix filter generating $\mathrm{CL}$ is symmetric, the construction of matrix filter is lack of universality. So we present a general method to construct a lowpass matrix filter at first. For scalar wavelets, the highpass filter is determined by an automatic way from the lowpass filter but it often fails here. The reason is that the lowpass fliter are matrices and they can not commute [9]. A new construction procedure is needed for multiwavelets. So we give some simple formulas to construct the highpass filter. For the complexity of construction the lowpass and highpass fiters, we focus only on the orthogonality but omit the good frequency response. So a prefilter technique should be presented to get a good combining frequency property of matrix filter together with prefilter.

In order to evaluate the performance of multiwavelets for image coding at low bit rate, efficient SPIHT coding of multiwavelet coefficients has been realized, accomplished with a suitable scanning strategy across scales and inside each detail subband.

\section{SYMMETRIC MATRIX FILTER}

Let $N_{1}, N_{2}, \ldots, N_{r}$ be a multiwavelet system and compactly support $\boldsymbol{L}_{2}(\boldsymbol{R})$ functions, and

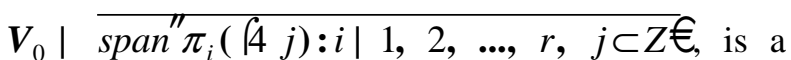
close space. Then $\boldsymbol{V}_{0}$ is called a finitely generated shift in variant space. Let $\left(\boldsymbol{V}_{p}\right)_{p \square z}$ be given by $\boldsymbol{V}_{p} \quad\left\{M 2^{p} \square: M \square \boldsymbol{V}_{0}\right\}$. The sequence $\left(\boldsymbol{V}_{p}\right)$ is 
called a MRA generated by $N_{1}, N_{2}, \ldots, N_{r}$ if (a) the spaces are nested $\ldots \boldsymbol{V}_{\square 1} \square \boldsymbol{V}_{0} \square \boldsymbol{V}_{1} \ldots$, and (b) the generator $N_{1}, N_{2}, \ldots, N_{r}$ and their integer translates form a Riesz basis for $\boldsymbol{V}_{0}$ [10]. Because of (a) and (b), we can write $\boldsymbol{V}_{j \square 1} \quad \boldsymbol{V}_{j} \square \boldsymbol{W}_{j}$, The space $\boldsymbol{W}_{0}$ is called multiwavelet space, and if $\left|{ }_{1},\right|_{2}, \ldots,\left.\right|_{r}$ generate a shift-invariant basis for $\boldsymbol{W}_{0}$, then these functions are called wavelet function.

Then (a) and (b) imply that $\boldsymbol{i j} \quad\left\{M, M_{2}, \ldots, M_{r}\right\}^{T}$ satisfies the dilation equation.

$$
\boldsymbol{i j}(x) \quad \mathbf{I}_{k} \boldsymbol{H}_{k} \mathbf{i j}(2 x \square k)
$$

The multiwavelets $\square \quad\left\{\left.\right|_{1},\left.\right|_{2}, \ldots,\left.\right|_{r}\right\}^{T}$ is given by

$$
\square(x) \quad \mathbf{I}_{k} \boldsymbol{G}_{k} \mathbf{i j}(2 x \square k)
$$

where $\boldsymbol{H}_{k}$ are a $r \mathrm{u} r$ matrix lowpass filter sequences and $\boldsymbol{G}_{k}$ are a $r \mathrm{u} r$ matrix highpass filter sequences. Let $\boldsymbol{H}(\boldsymbol{Z}) \mathbf{I}_{k} \boldsymbol{H}_{k} e^{\square j k Z / 2}$, $\boldsymbol{G}(Z) \quad \mathbf{I}_{k} \boldsymbol{G}_{k} e^{\square j k Z / 2}$, then Eq.(1) and Eq.(2) can be formulated in the Fourier domain

$$
\begin{aligned}
& \text { i }(Z) \quad \boldsymbol{H}(Z / 2) \check{\boldsymbol{i}}(Z / 2) \\
& \text { ] (Z) } \boldsymbol{G}(Z / 2) \check{\boldsymbol{i}}(Z / 2)
\end{aligned}
$$

For scalar wavelets, $\boldsymbol{G}_{k}$ are determined by an automatic way from $\boldsymbol{H}_{k}$ but it often fails here. The orthogonality of $\mathbf{i j}$ and $\square$ no longer follows identities like $\boldsymbol{H}_{0} \boldsymbol{H}_{1} \quad \boldsymbol{H}_{1} \boldsymbol{H}_{0}$, because $\boldsymbol{H}_{k}$ are matrices and they can not commute. Therefore a new construction procedure is needed for multiwavelets.

\section{Orthogonal conditions}

A scaling function $\boldsymbol{i j}$ is stable if and only if $\mathbf{I}_{k} \check{\mathbf{i}}(Z \square 2 k S) \check{\mathbf{i}}^{\square}(Z \square 2 k S)$ is positive definite for all $\angle$, and $\boldsymbol{i j}$ is orthogonal if and only if $\mathbf{I}_{k} \check{\boldsymbol{i}}(Z \square 2 k S) \check{\boldsymbol{i}}^{\square}(Z \square 2 k S) \quad \boldsymbol{I}_{r}$, and $\boldsymbol{H}_{k}$ and $\boldsymbol{G}_{k}$ are matrix filters. The orthogonal conditions now can be expressed as follows

$$
\begin{array}{cc}
\boldsymbol{H}(Z) \boldsymbol{H}^{*}(Z) \square \boldsymbol{H}(Z \square S) \boldsymbol{H}^{*}(Z \square S) & \boldsymbol{I}_{r} \\
\boldsymbol{G}(Z) \boldsymbol{G}^{*}(Z) \square \boldsymbol{G}(Z \square S) \boldsymbol{G}^{*}(Z \square S) & \boldsymbol{I}_{r} \\
\boldsymbol{H}(Z) \boldsymbol{G}^{*}(Z) \square \boldsymbol{H}(Z \square S) \boldsymbol{G}^{*}(Z \square S) & \boldsymbol{0}_{r}
\end{array}
$$

where $\left(\mathbb{D}^{*}\right.$ denotes complex conjugate transpose, where $\boldsymbol{I}_{r}$ and $\boldsymbol{O}_{r}$ are the $r \mathrm{U} r$ identity and null matrices, respectively. The orthogonal conditions Eq.(5), Eq.(6) and Eq.(7) are equivalent to

$$
\begin{aligned}
& \mathbf{I}_{k} \boldsymbol{H}_{k} \boldsymbol{H}_{k \square 2 j}^{T} \quad 2 \boldsymbol{G}_{j 0} \boldsymbol{I}_{r}, \quad j \square Z \\
& \mathbf{I}_{k} \boldsymbol{G}_{k} \boldsymbol{G}_{k \square 2 j}^{T} \quad 2 \boldsymbol{G}_{j 0} \boldsymbol{I}_{r}, \quad j \square Z \\
& \mathbf{I}_{k} \boldsymbol{H}_{k} \boldsymbol{G}_{k \square 2 j}^{T} \quad 0, \quad j \square Z
\end{aligned}
$$

The pair $\{\boldsymbol{H}, \boldsymbol{G}\}$ is called a multiwavelet filterbank. The matrix filters $\boldsymbol{H}, \boldsymbol{G}$ are called finite impulse response if there exists an integer $N$ so that $\boldsymbol{H}_{k} \quad 0, \boldsymbol{G}_{k} \quad 0,|k| ! N$.

\section{Construction of symmetric matrix filter}

In this subsection, we discuss the case $r 2$ and consider the situation where the first component of ij and $\square$ are symmetric, whereas the second components are antisymmetric. If $N_{i}$ is centrally antisymmetric for any odd number $i$ and centrally symmetric for any even number supported on $[0, L]$, then $\mathbf{i j}(x) \quad \boldsymbol{S} \mathbf{i j}(L \square x)$, where $\boldsymbol{S} \operatorname{diag}(1, \square 1)$. Using this in the dilation function Eq.(1), we obtain

$$
\text { ij } \quad \mathbf{I}_{k} \boldsymbol{H}_{k} \mathbf{i j}(2 \square k) \quad \boldsymbol{S i j}(L \square \square
$$




$$
\mathrm{I}_{k} \boldsymbol{S H}_{k} \boldsymbol{S i j}(2 \square k \square L) \quad \mathrm{i}_{k} \boldsymbol{S} \boldsymbol{H}_{L \square k} \boldsymbol{S i j}(2 \square k)
$$

If we assume $L \quad 2 N \square 1, N \square Z$, then $i \boldsymbol{j}$ will have the desired symmetric property provided that $\boldsymbol{H}_{k} \quad \boldsymbol{S H}_{2 N \square \square k} \boldsymbol{S}$. In this paper, we consider the case of $r \quad 2$, and $N \quad$ 2. We suppose the lowpass matrix filter $\boldsymbol{H}_{k}$ for scaling function is given by

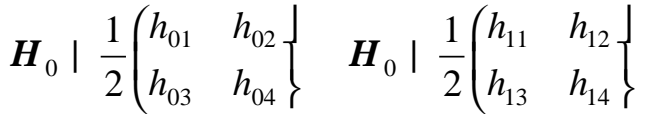

$$
\begin{aligned}
& \begin{array}{llll}
\boldsymbol{H}_{2} & \mathrm{SH}_{1} \boldsymbol{S}^{\prime} & \mathrm{H}_{3} & \mathrm{SH}_{0} \mathrm{~S}
\end{array}
\end{aligned}
$$

There exists a unique solution $\boldsymbol{i j}$ of the refinement equation such as $\check{\boldsymbol{i}}(0)\{1,0\}^{T}$. This solution is called the normalized solution. It was shown in [11] that $\boldsymbol{H}_{k}$ satisfies the order of vanishing moment condition at least one, i.e., there exists a nonzero 1u $r$ vector $\boldsymbol{M}$ such as $\boldsymbol{M H}(0) \boldsymbol{M}$, $\boldsymbol{M H}(S) \quad 0$. By Eq.(5), $\boldsymbol{M H}(0)^{T} \boldsymbol{M}$, thus $\boldsymbol{H}(0) \boldsymbol{M}^{T} \quad \boldsymbol{M}^{T}$, i.e., $\boldsymbol{M}^{T}$ is a right 1-eigenvector of $\boldsymbol{H}(0)$. By Eq.(3), $\check{\boldsymbol{i}}(0)$ is also a right 1-eignvector of $\boldsymbol{H}(0)$. Therefore, $\boldsymbol{M}^{T} \quad \check{\boldsymbol{i}}(0)$ since 1 is a simple eigenvalue of $\boldsymbol{H}(0)$. By Eq.(7), we have $\boldsymbol{M H}(0) \boldsymbol{G}(0)^{T} \square \boldsymbol{M H}(S) \boldsymbol{G}\left(S^{T}\right)^{T} \quad 0$, thus $\boldsymbol{M G}(0)^{T} \quad 0$, and hence $\boldsymbol{G}(0) \check{\boldsymbol{i}}(0) \quad 0$. By Eq.(4), we have $\square$ (0) 0. From Eq.(8), we have the frequency response of $\boldsymbol{H}_{k}$ at $\quad \boldsymbol{Z} \quad 0 \quad$ and $\angle S$ as follows

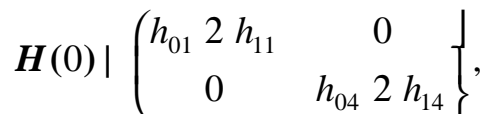

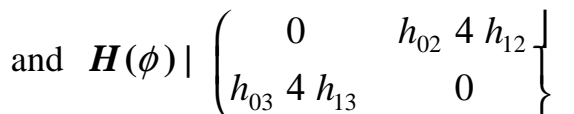

For example, the following $\boldsymbol{H}_{k}$ satisfies the orthogonal conditions Eq.(8).

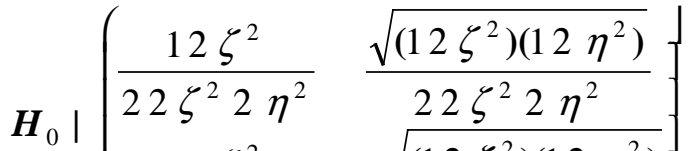

$$
\begin{aligned}
& \frac{\ll 1 \square D^{2}}{\ll 2 \square D^{2} \square E^{2}} \square \frac{\sqrt{\left(1 \square D^{2}\right)\left(1 \square E^{2}\right)} 》}{2 \square D^{2} \square E^{2}}{ }^{\Perp} / 4
\end{aligned}
$$

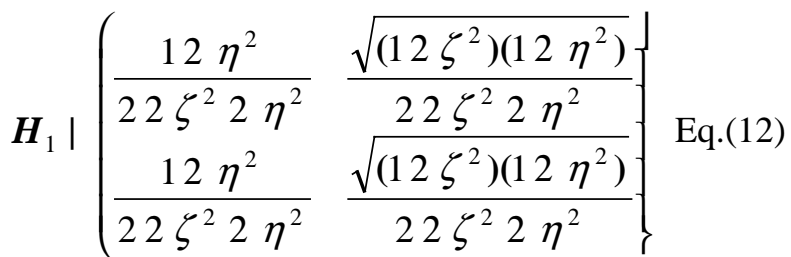

Let $\boldsymbol{G}_{k} \quad(\square 1)^{k} \boldsymbol{H}_{2 N \square \square k} \boldsymbol{P}$. If $\boldsymbol{H}_{k} \boldsymbol{P}_{2 N \square \square \square \square 2 i}^{T}$, $k \quad 0,1, \ldots, N \square i \square 1, i \quad 0,1, \ldots, N \square 1$, are symmetric matrix, then we have

$$
\begin{aligned}
& \left.\prod_{k 0}^{2 N \square 1 \square 2 i} \boldsymbol{H}_{k} \boldsymbol{G}_{k \square 2 i}^{T} \prod_{k 0}^{2 N \square 1 \square 2 i} \boldsymbol{H}_{k} \llbracket \square 1\right)^{k \square 2 i \square 1} \boldsymbol{P}_{2 N \square \square k \square 2 i}
\end{aligned}
$$

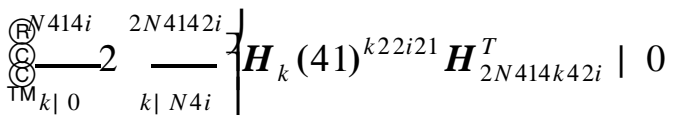

It is easy to verify that the $\boldsymbol{H}_{k}$ in Eq.(12) satisfies our previous hypotheses, so we can say that $\boldsymbol{H}_{k}$ and $\boldsymbol{G}_{k} \quad(\square 1)^{k} \boldsymbol{H}_{2 N \square 1 \square k} \boldsymbol{P}$ satisfy the orthogonal conditions Eq.(9) and Eq.(10).

\section{PREFILTER TECHNIQUE}

For the complexity of designing the matrix filter' we only consider the orthogonality but omit the good frequency property at first. By Eq.(13), we find $\boldsymbol{H}_{S O M}(Z)$ has a better frequency response than $\boldsymbol{H}_{G H M}(Z)$ and $\boldsymbol{H}_{C L}(Z)$, and so is $\boldsymbol{G}_{S O M}(Z)$. But as a whole, $\boldsymbol{G}_{S O M}(Z)$ is not a good highpass filter for $\boldsymbol{G ( 0 )}$ is not a 0 matrix. The decomposition result shown in Fig. 1(b) is not suitable for image compression. So we should present a prefilter technique to get good lowpass and higpass frequency properties. For GHM and CL, there are many researches having been done [12]-[14]. As illustrated in Fig.1(c), using the prefilter technique, we can get a good energy distribution after decomposition. A framework of decomposition and reconstruction 
combined with SOM and the corresponding prefilter is shown in Fig. 2.

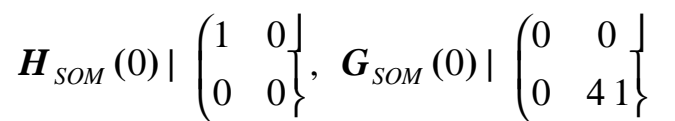

Let $A_{0}(Z)$ and $A_{1}(Z)$ be two components of the prefilter, and satisfy

$$
H_{00}(0) A_{0}(0) \square H_{01}(0) A_{1}(0) \quad 1
$$

$$
G_{10}(0) A_{0}(0) \square G_{11}(0) A_{1}(0) \quad 0
$$

By Eq.(13), we have $A_{0}(0) \quad 1$ and $A_{1}(0) \quad 0$. To ensure that we can reconstruct a decomposition signal completely, $A_{0}(Z)$ and $A_{1}(Z)$ should also satisfy the orthogonal condition

$$
A_{0}(Z) A_{0}^{*}(Z) \square A_{1}(Z) A_{1}^{*}(Z) \quad 1
$$

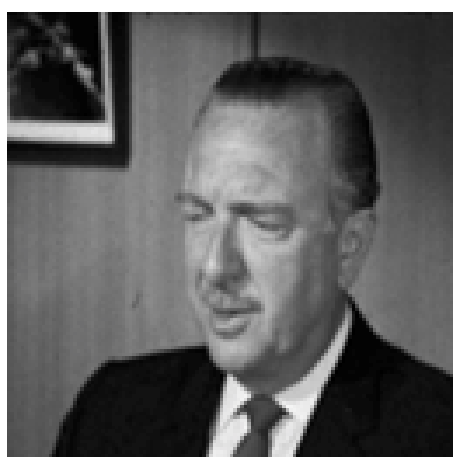

(a) Original

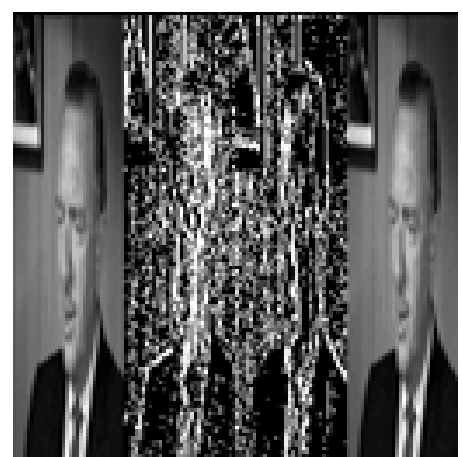

(b) Decomposition without prefilter

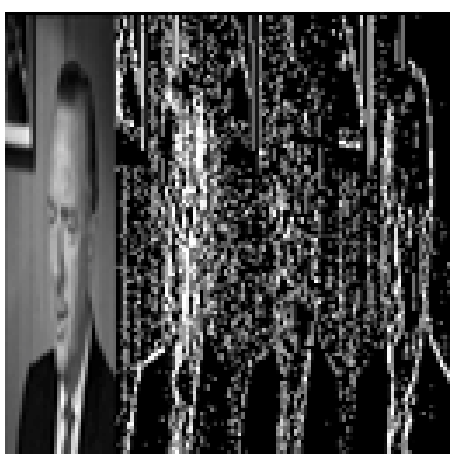

(c) Decomposition with prefilter

Fig. 1. Prefiltering can compact the energy in lowerpass subband

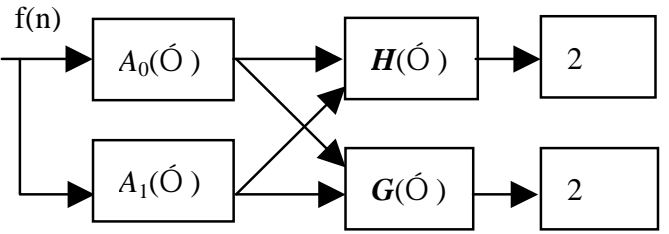

(a) Prefilter and decomposition

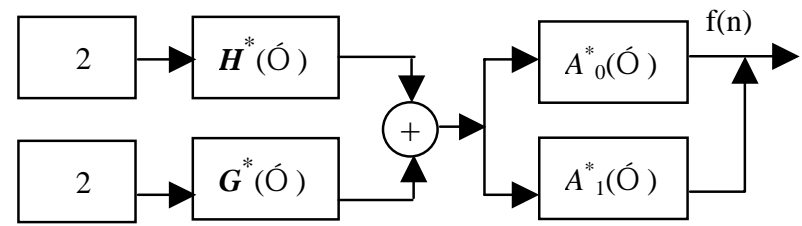

(b) Reconstruction and postfilter

Fig. 2. A framework of decomposition combining with SOM and prefilter.

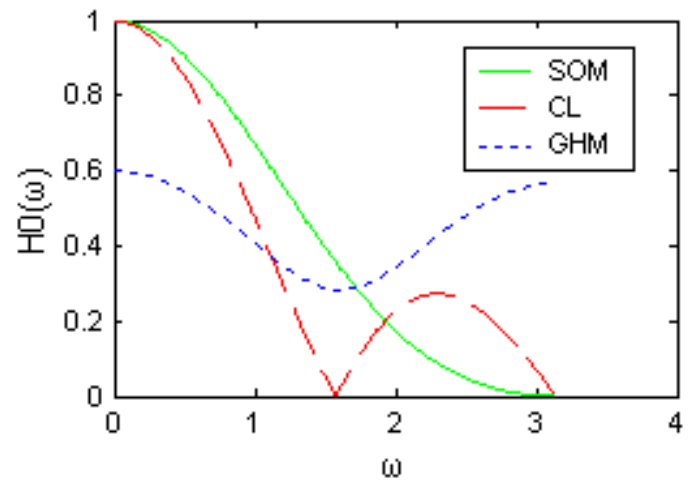

(a)

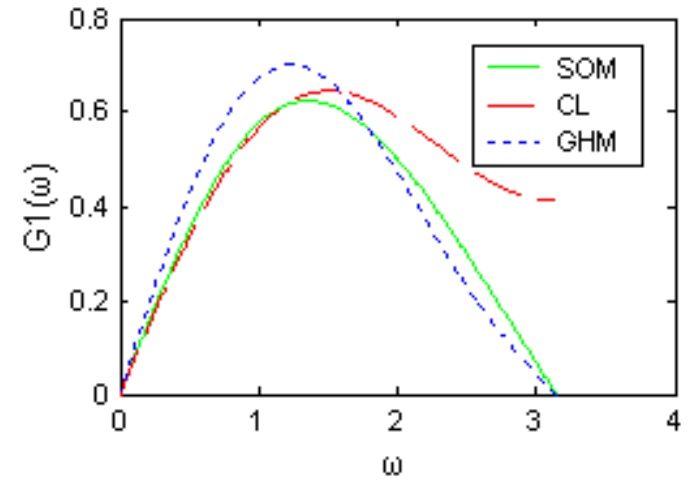

(b)

Fig. 3. Combining frequency response with Haar prefilter. (a) $\mathrm{H}_{0}\left({ }^{1}\right)$. (b) $\mathrm{G}_{1}\left({ }^{1}\right)$.

We find $A_{i}(Z) \quad 1 / 2 \square(\square 1)^{i} / 2 e^{\square j Z}$ is steady for both CL and SOM. Moreover, the computation complexity is lower than the prefilter presented in [12] and [13] for the parameters of prefilter are $1 / 2$ and $-1 / 2$ (We call it Haar prefilter). There are four 
combining frequency components as shown in Eq.(17) and Eq.(18). According to Eq.(17) and Eq.(18), the frequency responses of $\mathrm{H}_{0}\left({ }^{1}\right)$ and $\mathrm{G}_{1}\left({ }^{1}\right)$ are illustrated in Fig. 3.

$$
\begin{aligned}
& H_{C}(Z) \quad H_{C 0}(Z) A_{0}(Z) \square H_{C 1}(Z) A_{1}(Z) \\
& G_{C}(Z) \quad G_{C 0}(Z) A_{0}(Z) \square G_{C 1}(Z) A_{1}(Z)
\end{aligned}
$$

\section{SIMULATION RESULTS}

During a single level of decomposition using a scalar wavelets transform, a 2-D image data is replaced with four blocks corresponding to the subbands representing either lowpass or highpass filtering in each direction. The multiwavelets used here have two channels, so there will be two sets of scaling matrix coefficients and wavelets matrix coefficients. The first level multiwavelet decomposition subbands

\begin{tabular}{|l|l|l|l|}
\hline $\mathrm{L}_{1} \mathrm{~L}_{1}$ & $\mathrm{~L}_{1} \mathrm{~L}_{2}$ & $\mathrm{~L}_{1} \mathrm{H}_{1}$ & $\mathrm{~L}_{1} \mathrm{H}_{2}$ \\
\hline $\mathrm{L}_{2} \mathrm{~L}_{1}$ & $\mathrm{~L}_{2} \mathrm{~L}_{2}$ & $\mathrm{~L}_{2} \mathrm{H}_{1}$ & $\mathrm{~L}_{2} \mathrm{H}_{2}$ \\
\hline $\mathrm{H}_{1} \mathrm{~L}_{1}$ & $\mathrm{H}_{1} \mathrm{~L}_{2}$ & $\mathrm{H}_{1} \mathrm{H}_{1}$ & $\mathrm{H}_{1} \mathrm{H}_{2}$ \\
\hline $\mathrm{H}_{2} \mathrm{~L}_{1}$ & $\mathrm{H}_{2} \mathrm{~L}_{2}$ & $\mathrm{H}_{2} \mathrm{H}_{1}$ & $\mathrm{H}_{2} \mathrm{H}_{2}$ \\
\hline
\end{tabular}

(a)

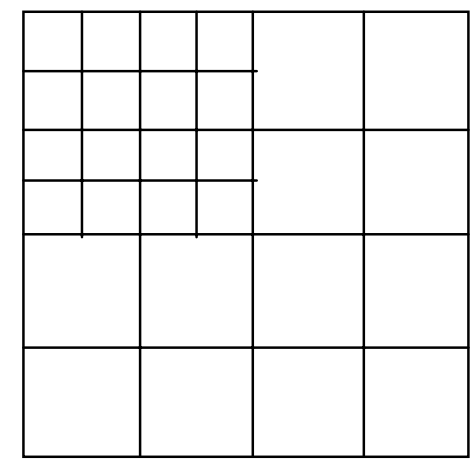

(c) and the relationship are shown in Fig.4(a) and (b).

Scalar wavelets transforms give a single quarter-sized lowpass subband from the original larger one. In previous literature, multiwavelet decompositions are performed in the same way. The multiwavelet decompositions iterate on the lowpass coefficients from the previous decomposition, the $\mathrm{L}_{\mathrm{i}} \mathrm{L}_{\mathrm{j}}$ subbands in Fig.4(a), as shown in Fig.4(c). In the case of scalar wavelets, the lowpass quarter image is a single subband. However, when the multiwavelet transform is used, the lowpass coefficients is actually a $2 \mathrm{~h} 2$ block of subbands, one lowpass and three bandpasses. Conventionally, the next decomposition step will decompose the lowpass subbands $\mathrm{L}=\left\{\mathrm{L}_{1} \mathrm{~L}_{1}, \mathrm{~L}_{1} \mathrm{~L}_{2}, \mathrm{~L}_{2} \mathrm{~L}_{1}, \mathrm{~L}_{2} \mathrm{~L}_{2}\right\}$. In this case, 2-level multiwavelet decomposition of a 2-D signal will produce $4(3 \mathrm{~h} 2+1)$ subbands.

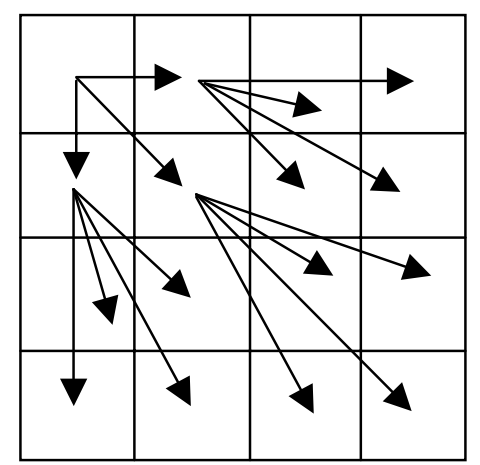

(b)

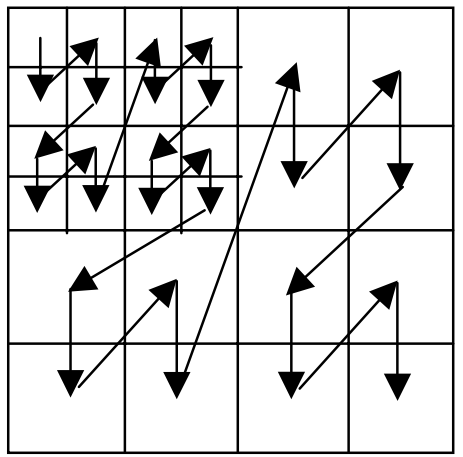

(d)

Fig. 4. (a) 4 subbands after first decomposition. (b) Parent-children relationship among the 4 subbands. (c) 28 subbands after second decomposition. (d) The traditional scan and quantization order among the 28 subbands.

The quantization method used to generate the result in this paper is the SPIHT [15]. SPIHT and other type of zero tree quantizers, such as SLCCA [16] and MRWD [17], achieve good performance by exploiting the spatial dependency of the pixels, which are in different subbands after scalar wavelet transform. The assumptions that the SPIHT quantizer makes about the relationship between subbands hold well for scalar wavelet, but they do not hold for multiwavelets for the multiwavelet transform 
destroys the parent-children relationship that SPIHT presumes as illustrated in Fig. 5(a). Then we present a new scan and quantization method that allows multiwavelet decompositions to receive most of the benefits from a SPIHT quantizer. The basic idea is to try to restore the spatial features that SPIHT requires for optimal performance. As a QMF-pyramid subband structure, the dependencies between ancestors and offsprings are show in Fig. 5(b).

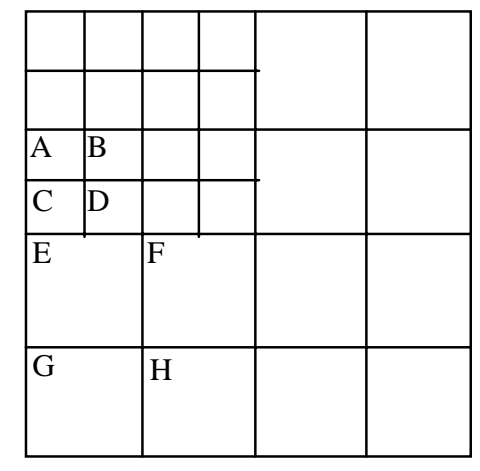

(a)

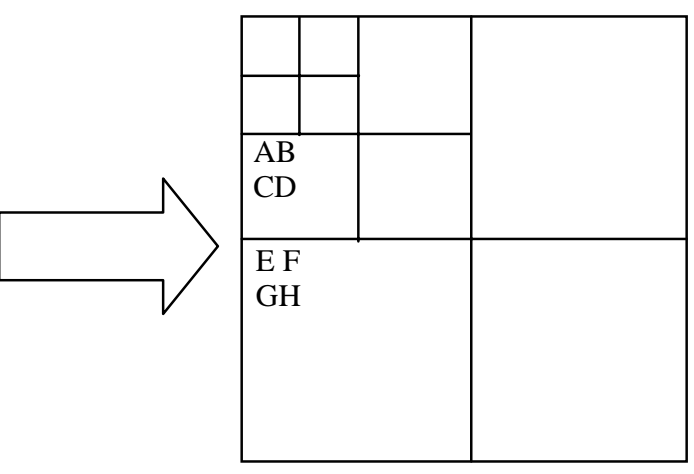

(b)

Fig. 5. Reorganizing the subband structure. (a) Before reorganization. (b) After reorganization, a scalar wavelet scan and quantization can be performed.

The new symmetric orthogonal multiwavelets, prefilter, and scan order are evaluated on four natural 512h 512 grayscale images, i.e., Lenna, Barbara, Boat, and Goodhill. For an accurate comparison, we have chosen a scalar wavelet for our experiment, an orthogonal and symmetric basis. Particularly, the following multiwavelets basis have been considered: GHM with the orthgonal approximation preserving prefilter, and CL with Haar transform matrix prefilter. Usually, the distortion is measured by peak signal to noise ratio.

Table I PSNR for compression of Lenna, Barbara, Boat, and Goodhill

\begin{tabular}{|c|c|c|c|c|c|c|c|c|c|}
\hline Image & Filter & $8: 1$ & $16: 1$ & $32: 1$ & Image & Filter & $8: 1$ & $16: 1$ & $32: 1$ \\
\hline \multirow{4}{*}{ Barbara } & $\mathrm{Bi} / 7 / 7$ & 35.84 & 31.67 & 27.37 & \multirow{4}{*}{ Goodhill } & $\mathrm{Bi} 9 / 7$ & 35.24 & 32.20 & 29.46 \\
\hline & GHM & 36.07 & 31.64 & 27.97 & & GHM & 35.80 & 32.35 & 29.87 \\
\hline & CL & 36.36 & 31.71 & 28.08 & & $\mathrm{CL}$ & - & 33.12 & 30.58 \\
\hline & SOM & 36.430 & 32.209 & 28.33 & & SOM & 36.43 & 33.211 & 30.473 \\
\hline \multirow{4}{*}{ Boats } & $\mathrm{Bi} / 7 / 7$ & - & - & - & \multirow{4}{*}{ Lenna } & $\mathrm{Bi} 9 / 7$ & 39.55 & 36.62 & 33.31 \\
\hline & GHM & 37.75 & 33.87 & 29.73 & & GHM & 40.14 & 37.70 & 33.30 \\
\hline & CL & $37.71-$ & 33.95 & 29.86 & & $\mathrm{CL}$ & 40.46 & 37.13 & 34.278 \\
\hline & SOM & 38.06 & 34.301 & 30.238 & & SOM & 40.64 & 37.791 & 34.478 \\
\hline
\end{tabular}

Table I shows the PSNR comparison on Lenna, Boat, Goldhill, and Barbara image at different bit rates. Our coder consistently outperforms Bi9/7. Compared to $\mathrm{Bi} 9 / 7$, our coder gains $0.15 \mathrm{~dB}$ to $0.25 \mathrm{~dB}$ in PSNR on average. Then we only compare the results that come from our coder with GHM. For Barbara image, our coder gains more than $0.5 \mathrm{~dB}$ at compression ratio (CR) 16:1, and gains $0.3 \mathrm{~dB}$ at compression ratio 8:1. For boat image, our coder gains $0.25 \mathrm{~dB}$ at $\mathrm{CR} 8: 1,0.45 \mathrm{~dB}$ at $\mathrm{CR} 16: 1$, and only gains $0.4 \mathrm{~dB}$ at $\mathrm{CR} 32: 1$. However, For the Goodhill and Lenna, which are relatively smooth images, the performance between our coder and
GHM gets closer. These preliminary results suggest that the SOM and prefilter for our coder is worthy of further investigation as a technique for complex textured image compression.

It is also noteworthy that symmetric multiwavelets can achieve good compression performance even though it has lower approximation order than both GHM and CL. So we can draw a conclusion that the approximation order and regularity are very important for some applications such as digital signal processing applications, but in image compression, the effect of approximation order and regularity is still unknown. 


\section{CONCLUSIONS}

Multiwavelets are an important development of wavelet theory for it solved the conflict between orthogonality and linear phase. Multiwavelets offer the advantages of combining symmetry, orthogonality, and short support, properties not mutually achievable with scalar wavelet system. For the special frequency response of the matrix filter, we introduce a new prefilter technique. In order to evaluate the performance of multiwavelets for image coding at low bit rate, efficient SPIHT coding of multiwavelets coefficients has been realized, accomplished with a suitable scanning strategy across scales and inside each detail subbimage. Extensive experimental results demonstrate that the parent-children relationship is much natural between finer and coarser scales multiwavelets coefficients, and our techniques exhibit performance equal to, even in several cases superior to the conventional scan and quantization methods.

\section{REFERENCES}

[1] Goodman T. N. T, Lee S. L, and Tang, "Wavelets in wandering subspaces" Trans. Amer. Math. Soc, 1993, Vol. 338, No. 1: 639-654.

[2] Goodman T. N. T and Lee S. L, "Wavelets of multiplicity r," Trans. Amer. Math. Soc, 1994, Vol. 342, No. 1: 307-324.

[3] Jia R. Q, Remeschneider S, and Zhou D. X, "Vector subdivision schemes and multiple wavelet" Elsevier Math. Comp, 1998, 67: 1533-1563.

[4] Chui C.K and Lian J, “A study on orthonormal multiwavelets," Appl. Numer. Math, 1996, Vol. 20: 273-298.

[5] Hong D and Wu A, "Orthgonal multiwavelets of multiplicity four" Elsevier Math.Comp, 2000, 40: 1153-1169.

[6] Tham J. Y, Shen L, and Lee S. L, “A general approch for analysis and application of discrete multiwavelet tansforms” IEEE Trans. Signal Processing, 2000, Vol. 48, No. 2: 457-464.

[7] Strela V and Heller P.N, "The application of multiwavelet filterbanks to image processing," IEEE Trans. Image Processing, 2000, Vol.8, No.4: .548-563, 1999.

[8] Cotronei M, Lazzaro D, Montefusco L. B, and Puccio L, "Image Compression Through Embedded Multiwavelet
Transform Coding,” IEEE Trans. Image Processing, 2000, Vol. 9, No. 2: 184-189.

[9] Strang G and Strela V, "Short wavelets and matrix dilation equations," IEEE Trans. Signal Processing, 1995, Vol. 43: 108-115.

[10] Donivan G. C, Geronimo J. S, and Hardin D. P, "Orthogonal polynomials and the contruction of piecewise polynomial smooth wavelets" Siam J. Math. Anal, 1999, Vol. 30, No. 5: 1029-1056

[11] Jiang Q, "Orthogonal multiwavelets with optium time-frequency resolution,” IEEE Trans. Signal Processing, 1998, Vol. 46, No.6: 830-844.

[12] Xia X. G, "A new prefilter design for discrete multiwavelet transforms," IEEE Trans. Signal Processing, 1998, Vol. 46, No.4: 1558-1570.

[13] Xia X. G, Geronimo J. S, and Hardin D. P, "Design of prefilters for discrete multiwavelet transforms," IEEE Trans. Signal Processing, 1996, Vol. 44, No. 1: 25-35.

[14] Hardin D.P and Roach D. W, "Multiwavelet prefilter I: Orthogonal prefilters preserving approximation order p d 2," IEEE Trans. Circuits Syst. II, 1998, Vol. 45, No.8: 1106-1112.

[15] A. Said, W.A.Pearlman. "A new fast and efficient image codec based on set partitioning in hierachical trees", IEEE Transactions on Circuits and Systems for Video technology, 6 (3): 243-250, 1996

[16] Bing-Bing Chai, Jozsef Vass, and Xinhua Zhuang. "Sinificance-linked connected component analysis for wavelet image coding", IEEE Transactions on Image Processing, 1999, 8(6): 774-783

[17] S. D. Servetto, K. Ramchandran, and M.T.Orchard, "Image coding based on a morphological representation of wavelet data", IEEE Trans. Image Processing, 8(9), pp. 1161-1174. 1999. 